\title{
MOTOR TRAFFIC INJURIES IN SUB-SAHARAN AFRICA
}

\author{
Hipolite Thomas Tarimo, Kilimanjaro Christian Medical College-Tanzania \\ DMSJ 2012; 19: 13-16 http://dx.doi.org/10.4314/dmsj.v19i1.3
}

\begin{abstract}
Introduction: Motor traffic injuries are recognized as a major public health problem in developing countries. MTIs continue to cause morbidity, mortality and disability in Sub Saharan Africa (SSA).
\end{abstract}

Objectives: To describe the magnitude of MTI's in SSA and to find the magnitude of morbidity and mortality caused by MTI's.

Methodology: The World Health Organization (WHO), PUBMED and EMBASE databases were used to identify documents and articles on MTIs in SSA and its effects. The literature review was supplemented by document review and interviews with a key informant in one region of Tanzania to determine the magnitude of morbidity and mortality associated with MTI's in Tanzania.

Results: The mortality rate due to MTI's in Lower and Middle Income Countries(LMIC's) including SSA is about 20 per 100,000 population while in High Income Countries(HIC's) it is about 10.3 per 100,000 population. MTI's also represent the ninth leading cause of Disability Adjusted Life Years (DALY's) lost and are forecasted by WHO to be the third leading cause of DALY's lost by 2020. Total number of reported accidents in Kilimanjaro region in 2008 was 906 while in 2009 it was 1,125 accounting for an increase of $24 \%$. The total mortality reported in 2008 was 147 and 202 in 2009 with an increase of 37\%. The total morbidity was 622 in 2008 and 933 in 2009 accounting for a $50 \%$ increase.

Discussion: The high mortality rate in SSA of about 20 per 100,000 population is contributed mainly by human factors with the major being reckless driving and negligence of drivers, pedestrians and passengers. Morbidity and mortality due to MTI's is increasing gradually over time as seen from the Kilimanjaro documents. The burden of MTI morbidity and mortality is predicted to rise by $80 \%$ in SSA and fall by $30 \%$ in HIC's by 2020 .

Conclusion \& recommendations: MTI is still a public health problem in LMIC's. Measures to improve human factors in causing MTA are urgently required in order to reverse the trend. I hereby conclude with the motto by the National Road Safety Council of Tanzania which says "Road safety is NO ACCIDENT" and that "Road safety is for sharing".

Corresponding author: Hipolite T.T. Address: P.O BOX 10398 ARUSHA, Telephone number: +255 753642 882, E-mail: hipolitethomas@yahoo.com 


\section{INTRODUCTION}

Motor traffic injuries are recognized as a major public health problem in developing countries. MTI's continue to cause morbidity, mortality and disability in Sub Saharan Africa (SSA).The causes of MTIs are categorized into three main aspects; Human factors: These include reckless driving, excessive speed, overtaking errors, and alcohol above a blood level of $0.05 \mathrm{~g} / 100 \mathrm{ml}$, negligent pedestrians, passengers, cyclists and cart pushers. External factors: Poor road condition. Bad surfaces, for example, cause the vehicle to be driven erratically in its direction, which also reduces the safety of brakes, steering and tyres. Lack of road signs and markings confuses drivers about direction or speed limitation especially on road curvatures. Poor road engineering and alignment like having narrow width which makes it difficult to overtake especially long trucks and cars with broad width. Vehicle condition: Poor mechanical condition of vehicles like nondurable tyres, poor body work, defective brakes and loose wheel nuts $[4,7]$.

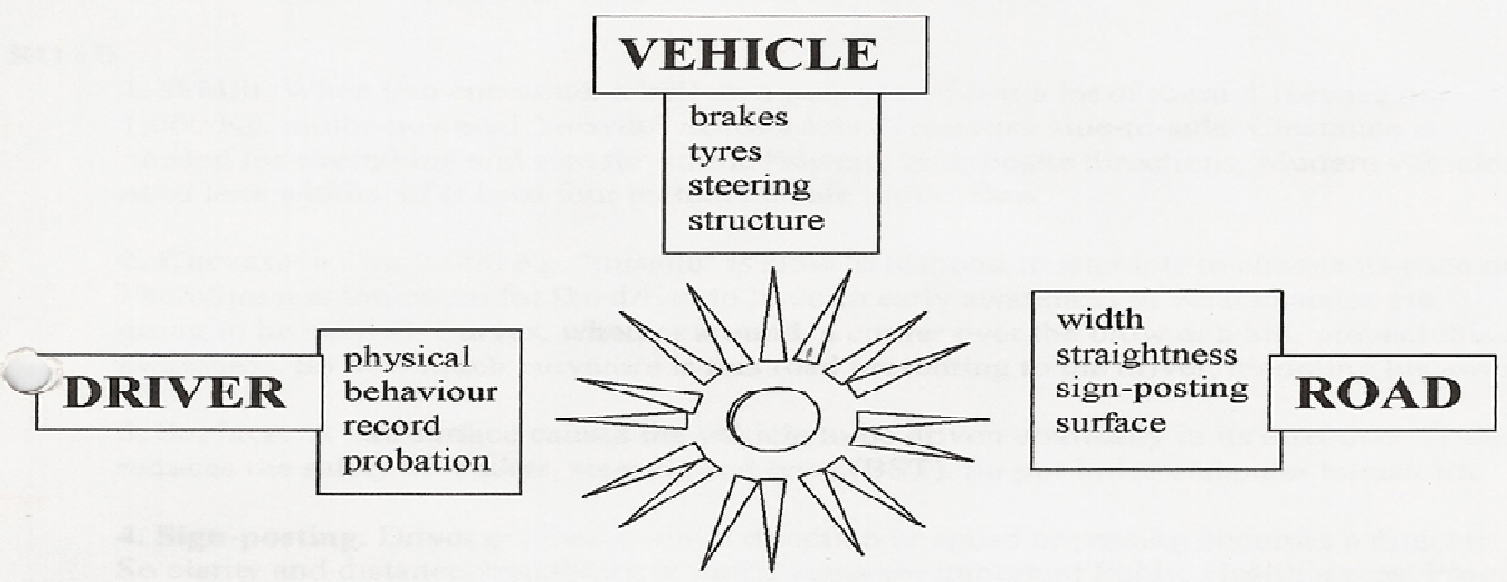

$$
\begin{aligned}
& \text { about vehicles } \\
& \text { about roads } \\
& \text { about drivers } \\
& \text { about national } \\
& \quad \text { dev. policy }
\end{aligned}
$$

RULES

Fig 1. Shows the interrelationship between MTI causative factors [9]

The objectives of this study were; a) to determine the burden of morbidity and mortality due to MTI's on Health Care Systems in SSA b) to describe the magnitude of morbidity and mortality among road users caused by MTI's c) to identify the vulnerable group among road users affected by MTIs and d) to examine the socio-economic cost of MTI's in SSA. 


\section{METHODOLOGY}

The World Health Organization (WHO), PUBMED and EMBASE databases were used to get relevant articles, reviews and other documentation on MTIs. The search words/strategy used to get relevant articles were; motor traffic, accidents, morbidity, and mortality, SSA; Epidemics \& Burden, MTIs. In total 18 articles or reviews were found and 12 were included in this review.

Relevant reports from the World Bank and Transport Research Laboratory were also reviewed.

Desk based-document review of reports of motor traffic accidents which occurred in Kilimanjaro region was done at the regional traffic police in Moshi. All the MTA's which occurred in 2008 and 2009 were reviewed to get the number of accidents occurring per year with associated outcomes (morbidity and mortality). Qualitative method i.e. key informant interview was conducted with the Regional Traffic Officer (RTO) of Kilimanjaro, to get deeper understanding of the data and his views on causes of accidents in the region. The data collected was used to determine the magnitude of morbidity and mortality particularly in Kilimanjaro region of Tanzania. Data analysis was done using STATA (version 7). Bivariate analyses were performed by cross tabulations and the chi-squared test was used to test for homogeneity. In the case of multiple injuries, the most recent injury episode was considered throughout the analysis.

The review also involved newspaper reports on motor traffic accidents occurring in Tanzania in 2010 .

\section{FINDINGS}

\section{MORBIDITY AND MORTALITY DUE TO MTIS}

The mortality rate due to MTI's in Lower and Middle Income Countries(LMIC's) including SSA is about 20 per 100,000 population while in High Income Countries (HIC's) it is about 10.3 per 100,000 population [4].

MTI's currently represent the ninth leading cause of Disability Adjusted Life Years (DALY's) lost and it is forecasted by WHO to be the third leading cause of DALY's lost by 2020 [4].

Mortality due to MTI's is predicted to increase by $80 \%$ in LMIC's and to decline by $30 \%$ in HIC's by 2020 [6].

The burden of morbidity and mortality for Traumatic Brain Injury (TBI) due to MTIs demonstrates a higher rate of 170 per 100,000 population in SSA compared to a global rate of 106 per $100,000[4]$.

The mortality per 10,000 vehicles is high in SSA (about 2000) as well as injuries per 10,000 vehicles (i.e. 16,000). These data values exceed those of HIC's like the United States as shown in fig 2 below [2]. 


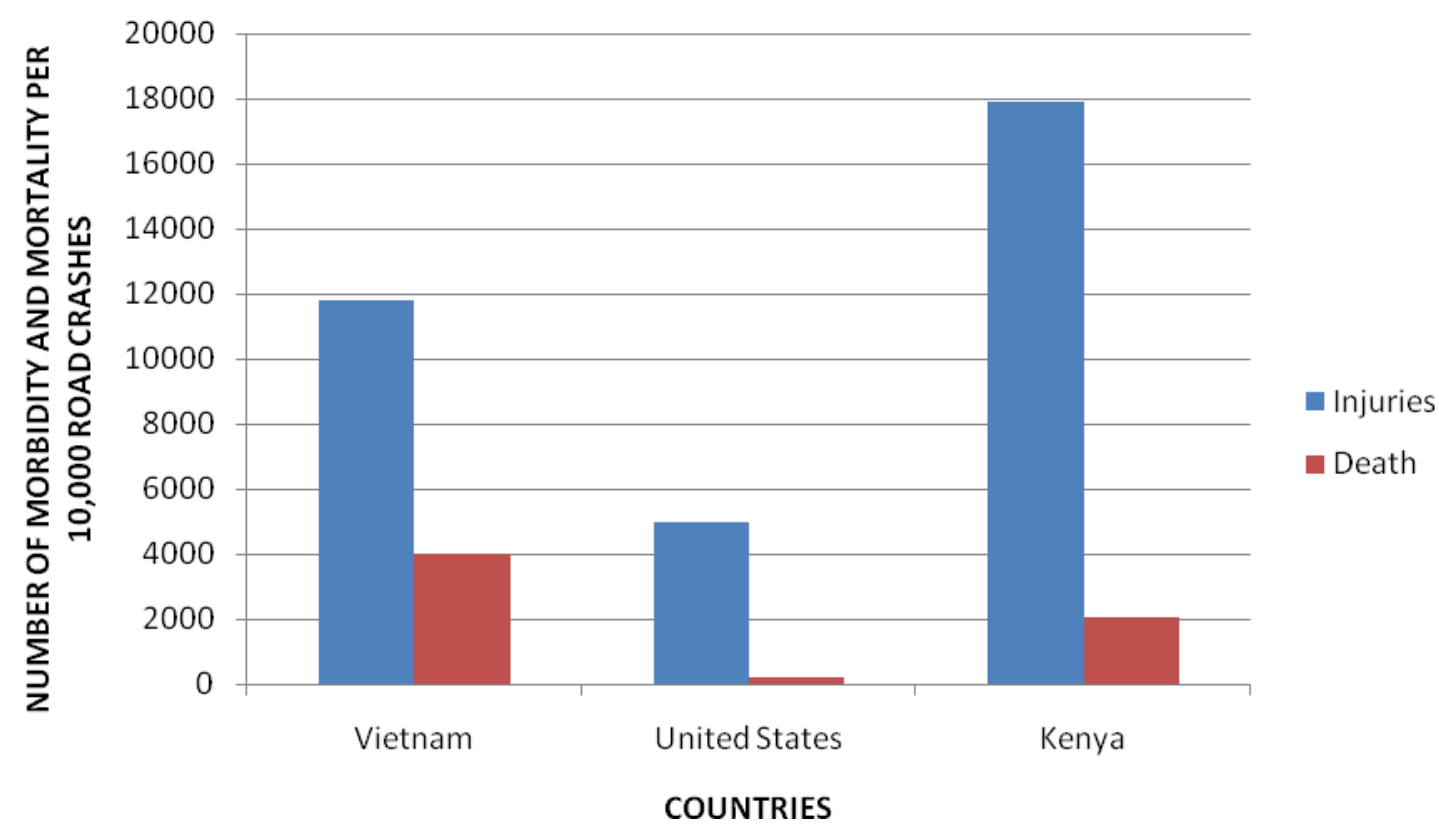

Fig 1. Comparison of morbidity and mortality per 10,000 vehicles between HIC's and LMIC's PREVALENCE OF MTA'S AND ASSOCIATED MORBIDITY IN TANZANIA

In Tanzania, there were a total of 11,223 accidents from January to June 2010 of which 2,085 involved motor cycles thus accounting for $18.6 \%$. The total number of deaths due to MTI's was 1,492 of which 286 involved motor cycles accounting for $19.2 \%$ of overall mortality [3]. Total number of reported accidents in Kilimanjaro region in 2008 was 906 while it was 1,125 in 2009 accounting for an increase of 24\%. The total mortality reported was 147 in 2008 and 202 in 2009 with an increase of $37 \%$ and the total morbidity was 622 in 2008 and 933 in 2009 accounting for a 50\% increase as shown in table $\mathbf{1}$ below [5].

Table 1: Morbidity and mortality due to MTIs in Kilimanjaro region within 2008/2009

\begin{tabular}{|c|c|c|c|}
\hline YEAR & 2008 & 2009 & \% INCREASE \\
\hline $\begin{array}{c}\text { TOTAL NUMBER OF } \\
\text { ACCIDENTS }\end{array}$ & 906 & 1,125 & 24 \\
\hline TOTAL MORTALITY & 147 & 202 & 37 \\
\hline
\end{tabular}




\begin{tabular}{|l|l|l|l|}
\hline TOTAL MORBIDITY & 622 & 933 & 50 \\
& & & \\
\hline
\end{tabular}

\section{VULNERABLE GROUPS AMONG ROAD USERS}

Pedestrians, cyclists and passengers are vulnerable groups among road users with a high risk of mortality due to MTI's i.e. about $46 \%$ in SSA which is contrary to the situation in HIC's. See the fig 3 below [4].

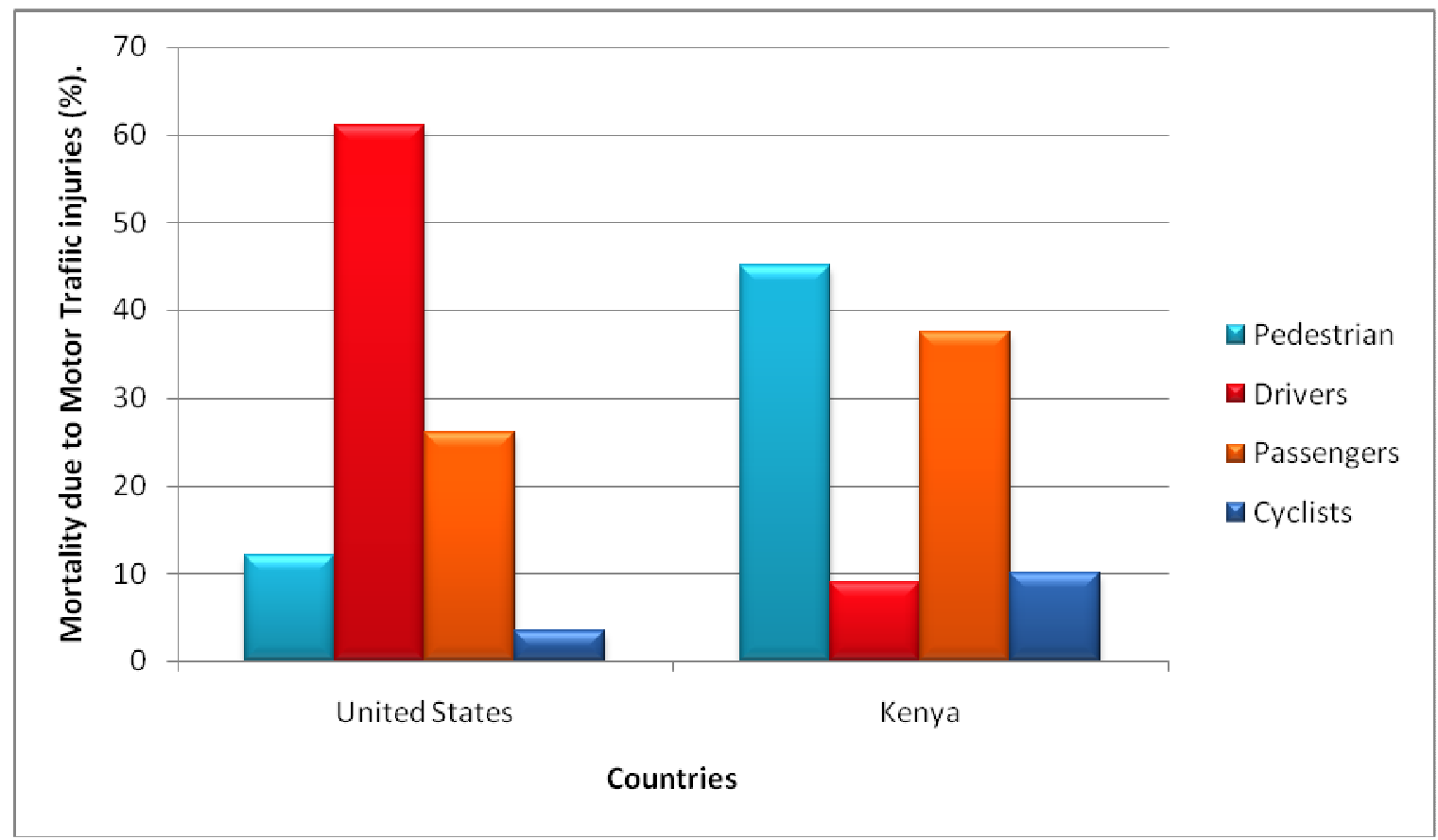

Fig 2.Mortality due to motor traffic among road user's in SSA and HICs [4]

MTI's are also predicted to become the top cause of Disability and premature death for children aged 5-14 years in LMIC's by 2015 [4].

\section{SOCIO-ECONOMIC IMPACT OF MTIs}

Globally the economic cost of MTI's is about US\$ 518 Billion and about US\$ 65 Billion in LMIC's [12]. The annual cost for road crashes is about $1 \%$ of Gross National Product (GNP) in developing countries and $2 \%$ in highly motorized countries [1]. 


\section{DISCUSSION}

The high mortality rate in SSA of about 20 per 100,000 population observed so far is contributed mainly by human factors with the major being reckless driving and negligence of drivers, pedestrians and passengers [7].

The percentage increase of morbidity and mortality due to MTI's is increasing gradually over time as shown through the recent years from table 1 above. This agrees with the prediction of mortality due to MTI's increasing by $80 \%$ in SSA by 2020 [6].

An increasing number of vehicles per inhabitant has not been a solution to MTI's in SSA. This is seen in the case of South Africa, the most developed African country having about 17 licensed vehicles per 100 inhabitants in 2005 yet NO decline of mortality due to MTI's has been observed so far [8].

The high burden of mortality from Traumatic Brain Injury (TBI) due to MTI's in SSA is found to be caused mainly by intracranial hemorrhage as shown by autopsies carried out on dead victims to ascertain the cause of death for legal procedures.

The major factor which will increase child pedestrian disability as a vulnerable group among road users by 2015 is lack of/poor adult supervision on those children aged 5-14 years who poorly adhere to the road safety rules.

The road crash annual cost expressed as a percentage of GNP provides a useful albeit crude approach to costing accidents, particularly on a global basis. But there is no real substitute in individual SSA countries for carrying out a detailed appraisal of national accident cost thus failing to pursue its social welfare objectives like minimizing of injuries or mortalities related to motor traffic.

\section{CONCLUSION}

MTI's are among the major causes of morbidity and mortality in LMIC's. The major contributing factor is the human factor with pedestrians, cyclists and passengers being the most vulnerable groups. I hereby conclude with the Motto by the National Road Safety Council of Tanzania which says "Road safety is NO ACCIDENT" and that "Road safety is for sharing" [10].

\section{RECOMMENDATIONS}

At Individual Level, the drivers should be aware of their physical senses i.e. sight, hearing and proprioception before they drive. Also they should have a sense of respect for others and for their own lives and above all they should not miss the sense of danger to their lives and others which is vital in the prevention of MTI's. Passengers should wear their seatbelts while in vehicles. Pedestrians should be aware of all the road traffic signs and follow the road safety rules. At Family Level, the parents should take time to educate their children about road safety rules and 
provide necessary supervision especially for those children aged 5-14 years. This will help to prevent the predicted increase in child pedestrian disability and premature death by 2015. (WHO, 2009a). At National Level, since the major contributing factor to MTI's in SSA has been found to be human factors mainly reckless driving, negligence of drivers, pedestrians, cyclists and passengers, stern measures should be implemented by the National Road Safety Council, Traffic Police and other responsible authorities, organizations and agencies to enforce the road safety laws and Acts, for example, The Road Traffic Act of Tanzania of 1973 section 40 and 41 (Causes of bodily injury and death through reckless driving and carelessness) [11].

\section{ACKNOWLEDGEMENT}

First and foremost, I thank the Almighty Lord for giving me energy, wisdom, motive and attitude of data collecting, proving, compiling and finally writing the whole report. I am also sending my gratitude to the Kilimanjaro Regional Traffic Police, especially the Regional Traffic Officer (RTO), for their cooperation in my report preparation. I also thank Mr. Njau of Community Department Kilimanjaro Christian Medical Center (KCMC) and Mrs. Lucy Lyaruu of (Tanzania Pesticide Research Institute (TPRI) for their great support without forgetting Adinan Juma (Former Academic Minister of Tumaini University Students Organization (TUSO), Kilimanjaro Christian Medical College (KCM College), Mohamed Kazim Alwani (Secretary General TUSOKCM College), Mr. Solomon Yusuph and my College mates Anzibert, Othman, Francis, Kevin and Daudi Marisani for their encouragement and support in one way or another throughout the preparation of my report.

\section{REFERENCES}

1. Aeron Thomas and Astrop, (2000).Exploring the relationship between Road traffic injuries and economic development.

2. Burden of morbidity and mortality in High Income Countries and Lower and Middle Income Countries. Transport Research Laboratory Report, 2000.

3. Editorial Opinion: Morbidity and Mortality of Motor Traffic Injuries in Tanzania. Mtanzania Newsletter, $15^{\text {th }}$ August 2010, Pg 5.

4. http;//www.who.int/world.health day/2009/informaterials/worldreport/en. (Accessed 10 August 2010).

5. Kilimanjaro Regional Police Station, (2010). Morbidity and mortality of Motor Traffic Injuries in Kilimanjaro region.

6. Kopits E \& Cropper M, Accid Anal Prev, (2003). Traffic fatalities and economic growth: 37: 169-178.

7. Myers RA, Taljaard JJ, Penman KM. Alcohol and road traffic injury. S Afr Med J. 1977; 52:328-330.

8. Road Traffic Management Corporation. (2006). December 2005 road traffic report on number of registered, un-roadworthy and un-licensed vehicles, driving licenses, PrDPs, traffic volumes, speed, distance travelled and fatal crashes. Available:

http://www.arrivealive.co.za/documents/stats/2005part1a.pdf.(Accessed 22 July 2010). 
9. Sheffer R, (2000). Road traffic accidents lecture notes.

10. Tanzania Public Health Association,(2004). Motor traffic accidents in Tanzania.

11. T. Rwebangira, T. Pearce, DAC Maunder, (1999): Public Transport Safety in Tanzania.

12. World Bank Group

www.worldbank.org/depweb/english/modules/glossary.htm. (Accessed $11^{\text {th }}$ August, 2010) 